\title{
WHEN IS THE ITERATE OF A FORMAL POWER SERIES ODD?
}

\author{
BRUCE REZNICK \\ (Received 1 May 1977; revised 15 February 1979) \\ Communicated by E. Strzelecki
}

\begin{abstract}
The formal power series (fps) $f(z)=\sum_{i=1}^{\infty} a_{i} z^{i}$ is homozygous mod $k$ if $a_{i} \neq 0$ and $a_{j} \neq 0$ implies $i \equiv j \bmod k$. This generalizes even and odd fps. If $f$ is homozygous $\bmod k$ then all iterates of $f\left(f_{n}=f \circ f_{n-1}\right)$ are also homozygous mod $k$, but the converse is false-there are many non-odd fps $f$ for which $f(f(z))=z$. It is shown that if $f$ is not homozygous $\bmod k$ but $f_{n}$ is homozygous, then $f_{n r}(z)=z$ for some $r$. If all coefficients are real then, in fact, $f(f(z))=z$.
\end{abstract}

Subject classification (Amer. Math. Soc. (MOS) 1970): 39 A 05.

\section{Introduction}

A formal power series (fps) may be classified as even or odd if all exponents which appear are even or odd integers. Any iterate of an even or odd fps is itself an even or odd fps, but the converse is not true. For example, the fps

$$
f(z)=c^{-1} \sum_{i=1}^{\infty}(-c z)^{i}=-z(1+c z)^{-1}
$$

satisfies $f(f(z))=z$. Roughly speaking, this will turn out to be the only type of exception; if an fps function which is not odd (or even) has an iterate which is odd (or even), then some further iterate is $z$.

The work in this paper grew out of an NSF Summer Undergraduate Research Project on $f(f(x))=\sin x$. My advisor at that time was Professor W. A. J. Luxemburg, and I wish to express my general gratitude to him. I also wish to thank the referee for suggesting the germ of the final corollary as well as several simplifications of the proofs.

Author supported in part by an NSF undergraduate research grant. 


\section{Notation and preliminaries}

Throughout we shall consider formal power series with fixpoint 0 (that is, $f(0)=0$ ). All theorems may be easily recast to allow for different fixpoints. Iteration without fixpoints requires conditions on convergence which remove us from the realm of the fps.

An fps $f, f(z)=\sum_{i=1}^{\infty} a_{i} z^{i}, a_{i}$ complex, will be called homozygous mod $k$ if $a_{i} \neq 0, a_{j} \neq 0$ implies $i \equiv j \bmod k$. If $f$ is not homozygous $\bmod k$ it will be called hybrid $\bmod k$. Every fps is homozygous mod 1, even or odd fps are homozygous mod 2. The composition of the two fps $f$ and $g$ is written $f_{\circ} g$ and the iterates of $f$ are defined inductively by $f_{n}=f_{\circ} f_{n-1}$. If $a_{1} \neq 0$ then the coefficients of an fps $g$ satisfying $f(g(z))=z$ may be determined recursively; $g(z)=a_{1}^{-1} z+\ldots$ is written $f^{-1}(z)$. The fps $f$ and $g$ commute if $f \circ g=g \circ f$. Any two iterates of $f$ commute.

The first lemma is trivial but useful. The proof is omitted.

LEMMA 1. The following are equivalent.

(i) The fps $f(z)$ is homozygous mod $k$.

(ii) For some primitive $k$ th root of unity $\varepsilon$ and some integer $r, 0 \leqslant r \leqslant k-1$, $f(\varepsilon z)=\varepsilon^{r} f(z)$.

(iii) One can write $f(z)=\sum_{j=0}^{\infty} a_{j k+r} z^{j k+r}(1 \leqslant r \leqslant k)$.

LEMMA 2. If $f$ is homozygous mod $k$ then all iterates of $f$ are homozygous mod $k$.

PROOF. If $f(\varepsilon z)=\varepsilon^{r} f(z)$ then $f\left(\varepsilon^{m} z\right)=\varepsilon^{r m} f(z)$ by induction, hence

$$
f_{n}(\varepsilon z)=\varepsilon^{r^{n}} f(z) \text {. }
$$

The remainder of this paper is devoted to finding a converse for Lemma 2 . We shall need several results of Baker (1962). Baker's proofs are relatively short and will not be reproduced here.

THEOREM. (Baker (1962).)

(i) If $g$ and $h$ commute, where $g(z)=\sum_{i=1}^{\infty} a_{i} z^{i}$ and $h(z)=z+\sum_{i=m+1}^{\infty} b_{i} z^{i}$, $b_{m+1} \neq 0, m \geqslant 1$, then $a_{1}^{m}=1$.

(ii) If, in (i), $a_{1}=1$, then $a_{2}=\ldots=a_{m}=0$ while $a_{m+1}$ is arbitrary and to each $\lambda$, there is a unique fps $g$, denoted $h_{\lambda}$, with $a_{m+1}=\lambda b_{m+1}$.

(iii) For each $a_{1}$ with $a_{1}^{m}=1$, there is exactly one fps $H(z)=\sum_{i=1}^{\infty} c_{i} z^{i}$ with $c_{1}=a_{1}, H_{m}(z)=z$ and $H \circ h=h_{\circ} H$. The series $g(z)=\sum_{i=1}^{\infty} a_{i} z^{i}$ with $a_{1} z$ as leading term are precisely $H \circ h_{\lambda}$ for various $\lambda$. 


\section{Spadework}

Suppose $f(z)=\sum_{i=1}^{\infty} a_{n_{i}} z^{n_{i}}\left(a_{n_{i}} \neq 0\right)$ is a hybria fps $\bmod k$. Write $f \sim\left(n_{1}, n_{j}\right)_{k}$ where $j$ is the smallest index for which $n_{j} \not \equiv n_{1} \bmod k$.

LEMMA 3. If $f \sim(c, d)_{k}$ and $c>1$, then any iterate $f_{m}$ is also hybrid mod $k$ and $f_{m} \sim\left(c^{m}, c^{m}+d-c\right)_{k}$.

PROOF. Suppose $f(z)=\sum_{i=1}^{\infty} a_{n_{i}} z^{n_{i}}$ and $f_{s}(z)=\sum_{k=1}^{\infty} b_{m_{k}} z^{m_{k}}$ where $a_{n_{i}}, b_{m_{k}} \neq 0$. Then $f_{s+1}(z)=\sum_{i=1}^{\infty} a_{n_{i}}\left(\sum_{k=1}^{\infty} b_{m_{k}} z^{m_{k}}\right)^{n_{i}}$ and so $z^{r}$ will appear in $f_{s+1}(z)$ only if $r$ is a sum of $n_{i}$ terms each of which is an $m_{k}$. Even so, there may be cancellations: $f(z)=\sum_{i=1}^{\infty}(-1)^{i} z^{i}, f(f(z))=z$. The lemma will be proved by induction on $m$; the result is true for $m=1$. Assume it for $m=s$; then, keeping the previous notation, $n_{1}=c, m_{1}=c^{8}, m_{j^{\prime}}=c^{8}+d-c$ and $m_{i} \equiv m_{1} \bmod k$ for $1 \leqslant i<j^{\prime}$. The smallest exponent in $f_{s+1}$ will come from the smallest possible sum $\sum_{u=1}^{n_{1}} m_{k_{u}}$, which is clearly $c^{s+1}$. The coefficient of $z^{c^{+1}}$ derived from $f_{\circ} f_{s}$ is $a_{c}\left(b_{c}\right)^{c}$ which does not vanish. If $n_{i} \equiv n_{1}$ and each $m_{k_{u}} \equiv m_{1}$, then the sum is congruent to $c^{8+1}$. The smallest exponent not congruent to $c^{8+1}$ thus arises either when $n_{i} \not \equiv n_{1}$ and each $m_{k_{u}} \equiv m_{1}$ (giving $c^{8} d$ ) or when $n_{i}=n_{1}$, one $m_{k_{u}}$ is $m_{j}$, and the rest are $m_{1}$ (giving $\left.c^{s+1}+d-c\right)$. The latter is smaller and the coefficient of $z^{c^{s+1}+d-c}$ derived in this way is $c a_{c}\left(b_{c^{s}}\right)^{c-1} b_{c^{s}+d-c}$, which does not vanish. Accordingly, the induction hypothesis is verified for $m=s+1$.

LEMMA 4. Suppose $f(z)=\sum_{i=1}^{\infty} a_{n_{i}} z^{n_{i}}, a_{n_{i}} \neq 0$ and $f \sim(1, t)_{k}$. Then the coefficients of $z$ and $z^{i}$ in $f_{n}$ are $a_{1}^{n}$ and $a_{l} a_{1}^{n-1} \sum_{i=0}^{n-1}\left(a_{1}^{l-1}\right)^{i}$ respectively.

Proof. The arguments of the last lemma demonstrate that any power of $z$ less than $t$ which appears in $f_{n}$ is congruent to $1 \bmod k$. This lemma is also proved by induction on $n$ and is evidently true for $n=1$. Assume it true for $n=r$. We have $f(z)=\sum_{i=1}^{\infty} a_{n_{i}} z^{n_{i}}, n_{1}=1, a_{1} \neq 0, n_{j}=t, t \neq \equiv 1 \bmod k, n_{i} \equiv 1 \bmod k$ for $1 \leqslant i<j$ and $f_{r}(z)=\sum_{i=1}^{\infty} b_{m_{i}} z^{m_{i}}, m_{1}=1, b_{1}=a_{1}^{r}, m_{j^{\prime}}=t, m_{i} \equiv 1 \bmod k$ for $1 \leqslant i<j^{\prime}$. Also, $f_{r+1}(z)=f\left(f_{r}(z)\right)=a_{1} f_{r}(z)+\sum_{i=2}^{\infty} a_{n_{i}}\left(f_{r}(z)\right)^{n_{i}}$. If $d(s)$ is the coefficient of $z^{\prime}$ in $f_{s}(z)$, then $d(1)=a_{t}$ and $d(r)=a_{t} a_{1}^{r-1} \sum_{i=0}^{r-1}\left(a_{1}^{l-1}\right)^{i}$ by hypothesis. Considering how $z^{t}$ can appear in $f_{r+1}(z)$, we have

$$
d(r+1)=a_{1} d(r)+a_{l}\left(a_{1}^{r}\right)^{l}=a_{t} a_{1}^{r} \sum_{i=0}^{r-1}\left(a_{1}^{t-1}\right)^{i}+a_{l} a_{1}^{r l}=a_{l} a_{1}^{r} \sum_{i=0}^{r}\left(a_{1}^{l-1}\right)^{i}
$$

and the lemma is proved. 


\section{On the inheritance of the homozygous trait}

The following theorem is a converse to Lemma 2.

THEOREM. If $f$ is hybrid mod $k$ and $f_{n}$ is homozygous mod $k$, then $f_{n s}(z)=z$ for some integer s. Further, if $f(z)=\sum_{i=1}^{\infty} a_{i} z^{i}$, then $a_{1}$ is a primitive nsth root of unity. In particular, if $f$ is real, then $f(f(z))=z$.

Proof. Suppose $f \sim(c, t)_{k}$. In the light of Lemma 3, we must have $c=1$, and, by Lemma 4 , the coefficient of $z^{l}$ in $f_{n}$ is $a_{1}^{n-1} a_{t} \sum_{i=0}^{n-1}\left(a_{1}^{t-1}\right)^{i}$. As $f_{n}$ is homozygous $\bmod k$ and $t \equiv 1 \bmod k$, this coefficient must vanish. Hence, $\left(a_{1}^{l-1}\right)^{n}=1$ and $a_{1}^{-1} \neq 1$. Let $h=f_{n(l-1)} ; h$ is homozygous with leading term $a_{1}^{(l-1) n} z=z$. Let $\varepsilon$ be a primitive $k$ th root of unity and define $g(z)=\varepsilon^{-1} f(\varepsilon z)$. As $f$ is hybrid $\bmod k$, $f(\varepsilon z) \neq \varepsilon f(z)$, so $f \neq g$. The leading term of both $f$ and $g$ is $a_{1} z$. Since $f_{n}$ is homozygous, $f_{n}(\varepsilon z)=\varepsilon f_{n}(z)$ and so $g_{n}(z)=f_{n}(z)$. It follows that $f_{n(l-1)}=g_{n(l-1)}=h$.

Suppose that $h(z) \neq z$, then $h(z)=z+\sum_{n=m+1}^{\infty} b_{n} z^{n}, b_{m+1} \neq 0$ and $k$ divides $m$ since $h$ is homozygous. We now apply the several parts of Baker's theorem. Since both $f$ and $g$ commute with $h$ and have the same leading coefficient, there exists $H(z)=\sum_{i=1}^{\infty} c_{i} z^{i}$ and $h_{\lambda}(z)=z+\lambda b_{m+1} z^{m+1}+\ldots$ such that $f=H \circ h_{p}$ and $g=H \circ h_{q}$. By construction, the coefficient of $z^{m+1}$ in $H \circ h_{\lambda}$ is $\lambda c_{1} b_{m+1}+c_{m+1}$, but the coefficient of $z^{m+1}$ is the same in $f$ and $g$ because $m+1 \equiv 1 \bmod k$. Thus $p c_{1} b_{m+1}+c_{m+1}=q c_{1} b_{m+1}+c_{m+1}$, and, since $c_{1} b_{m+1} \neq 0, p=q$ so that $f=g$, a contradiction. Therefore, $h(z)=z$.

Finally, suppose $a_{1}^{r}=1$ for $r<n(t-1)$; we may take $r r^{\prime}=n(t-1), r^{\prime}>1$. Let $k=f_{r}$, then $k_{r}(z)=z$. If $k(z) \neq z$, then $k(z)=z+a_{t} z^{i}+\ldots a_{t} \neq 0$. By lemma 4 , $k_{r}(z)=z+r^{\prime} a_{l} z^{i}+\ldots \neq z$, a contradiction, so $f_{r}(z)=z$ in any case. In particular, if $f$ is real, then $a_{1}$ is real hence $a_{1}=-1$ and $f(f(z))=z$.

\section{Examples}

We may characterize the fps $f$ in the last theorem still further. Suppose $f_{r}(z)=z$ and $f(z)=\varepsilon_{r} z+\ldots$, where $\varepsilon_{m}$, in general, is an $m$ th root of unity. There is a canonical fps $T$ such that $f(z)=T^{-1}\left(\varepsilon_{r} T(z)\right)$. Indeed, let

$$
T(z)=r^{-1} \sum_{j=0}^{r-1} \varepsilon_{r}^{-j} f_{j}(z) ;
$$

$T(z)=z+\ldots$ is invertible. It is readily verified that $T(f(z))=\varepsilon_{r} T(z)$; this is a solution to the Schröder equation. Further, the expressions of $f$ and $T$ in terms of 
each other show that they are either both homozygous $\bmod k$ or both hybrid $\bmod k$. (Note that, if an fps $h$ is invertible and homozygous, then so is $h^{-1}$.)

COROLlARY. Using the previous notation, $f$ is hybrid mod $k$ and $f_{n}$ is homozygous $\bmod k$ (and so $f_{n s}(z)=z$ ) if and only if $f(z)=T^{-1}\left(\varepsilon_{n s} T(z)\right.$ ), where $T=R_{\circ} U$, $R$ is hybrid mod $k$ and homozygous $\bmod s$, and $U$ is homozygous $\bmod k$, and both $R$ and $U$ are invertible.

Proof. If $h$ is invertible and homozygous $\bmod k$ and $h_{1}=h_{\circ} h_{2}$, then $h_{2}=h^{-1} \circ h_{1}$ so that $h_{1}$ and $h_{2}$ are either both homozygous or both hybrid. Suppose first that $T, R$ and $U$ are as described and $f(z)=T^{-1}\left(\varepsilon_{n s} T(z)\right)$. By the previous argument, $T$ is hybrid $\bmod k$ and so $f$ is too. Let $\varepsilon_{s}$ be the coefficient of $z$ in $f_{n}(z)$; it is the sth root $\varepsilon_{n s}^{n}$. We have $f_{n}(z)=T^{-1}\left(\varepsilon_{n s}^{n} T(z)\right)=T^{-1}\left(\varepsilon_{s} T(z)\right)=U^{-1}\left(R^{-1}\left(\varepsilon_{s} R(U(z))\right)\right)$. Since $R$ is homozygous $\bmod s, \varepsilon_{s} R(U(z))=R\left(\varepsilon_{s}^{m}(U(z))\right)$, so $f_{n}(z)=U^{-1}\left(\varepsilon_{s}^{m} U(z)\right)$ and is homozygous $\bmod k$.

On the other hand, suppose $f$ is hybrid $\bmod k$ and $f_{n}$ is homozygous $\bmod k$. Define $T$ as before and let $U(z)=s^{-1} \sum_{j=0}^{s-1} \varepsilon_{s}^{-j} f_{n j}(z)$. Then $U$ is homozygous $\bmod k\left(\right.$ since $f_{n}$ is) and $f_{n}(z)=U^{-1}\left(\varepsilon_{s} U(z)\right)=T^{-1}\left(\varepsilon_{s} T(z)\right)$. Therefore,

$$
T\left(U^{-1}\left(\varepsilon_{s} U(z)\right)\right)=\varepsilon_{s} T(z) .
$$

Let $R=T_{\circ} U^{-1}$ and $z=U^{-1}(y)$, then $R\left(\varepsilon_{s} y\right)=\varepsilon_{s} R(y)$, so that $R$ is homozygous $\bmod s$. Since $T$ is hybrid and $U$ is homozygous $\bmod k, R$ is hybrid $\bmod k$.

\section{Reference}

1. N. Baker (1962), 'Permutable power series and regular iteration', J. Austral. Math. Soc. 2, 265-294.

Department of Mathematics

University of California

Berkeley, California 94720

U.S.A. 